\title{
Two-photon photoemission from a copper cathode in an $X$-band photoinjector
}

\author{
H. Li, ${ }^{*}$ C. Limborg-Deprey, C. Adolphsen, D. McCormick, M. Dunning, K. Jobe, \\ T. Raubenheimer, A. Vrielink, T. Vecchione, F. Wang, and S. Weathersby \\ SLAC National Accelerator Laboratory, Menlo Park, California 94025, USA
}

(Received 16 July 2015; published 24 February 2016)

\begin{abstract}
This paper presents two-photon photoemission from a copper cathode in an $X$-band photoinjector. We experimentally verified that the electron bunch charge from photoemission out of a copper cathode scales with laser intensity (I) square for $400 \mathrm{~nm}$ wavelength photons. We compare this two-photon photoemission process with the single photon process at $266 \mathrm{~nm}$. Despite the high reflectivity $(R)$ of the copper surface for $400 \mathrm{~nm}$ photons $(R=0.48)$ and higher thermal energy of photoelectrons (two-photon at $200 \mathrm{~nm}$ ) compared to $266 \mathrm{~nm}$ photoelectrons, the quantum efficiency of the two-photon photoemission process $(400 \mathrm{~nm})$ exceeds the single-photon process $\left(266 \mathrm{~nm}\right.$ ) when the incident laser intensity is above $300 \mathrm{GW} / \mathrm{cm}^{2}$. At the same laser pulse energy $(E)$ and other experimental conditions, emitted charge scales inversely with the laser pulse duration. A thermal emittance of $2.7 \mathrm{~mm}$-mrad per mm root mean square (rms) was measured on our cathode which exceeds by sixty percent larger compared to the theoretical predictions, but this discrepancy is similar to previous experimental thermal emittance on copper cathodes with $266 \mathrm{~nm}$ photons. The damage of the cathode surface of our first-generation $X$-band gun from both rf breakdowns and laser impacts mostly explains this result. Using a $400 \mathrm{~nm}$ laser can substantially simplify the photoinjector system, and make it an alternative solution for compact pulsed electron sources.
\end{abstract}

DOI: 10.1103/PhysRevAccelBeams.19.023401

\section{INTRODUCTION}

Single-photon photoemission is the standard photoemission process used in electron guns for both metallic [1] and semiconductor cathodes [2]. One usually chooses a laser wavelength such that the energy of a single photon just exceeds work function of the cathode material. Compared to semiconductor cathode, copper cathodes can sustain very high gradient electric fields, above $200 \mathrm{MV} / \mathrm{m}$, survive poor vacuum conditions down to $10^{-7}$ torr scale, and have long lifetimes. Copper cathodes in radio frequency (rf) guns can recover from damage and pollution respectively by rf processing and laser cleaning. The copper cathode in the S-band gun of the Linac Coherent Light Source has been in use $24 / 7$ for the past several years, the copper cathode in the $X$-band gun at this facility has been used for two and a half years. The assets of metallic cathodes are unfortunately counterbalanced by its flaws, particularly its low quantum efficiency $(\mathrm{QE})$ of normally $10^{-5}$, and the necessity of using UV light. The low QE requires high pulse energy. This also prevents using copper cathodes in high repetition-rate electron guns, because the thermal

\footnotetext{
*Corresponding author. hl344@cornell.edu
}

Published by the American Physical Society under the terms of the Creative Commons Attribution 3.0 License. Further distribution of this work must maintain attribution to the author $(s)$ and the published article's title, journal citation, and DOI. effects deteriorate its performance and even cause damage when operating at $\mathrm{kHz}$ to tens of $\mathrm{kHz}$ repetition rates.

We experimentally studied the two-photon emission on a copper cathode $[3,4]$ to determine the adequacy of using a $400 \mathrm{~nm}$ wavelength, with a view to simplifying laser systems and a possibility of increasing the QE [5-8].

The work function $\varphi$ of bulk copper is approximately 4.5 to $5.1 \mathrm{eV}$ (we use $4.7 \mathrm{eV}$ in this paper), it requires ultraviolet (UV) photons and rf fields to overcome its work function to generate photoelectrons with single photons. The emitted charge is linearly proportional to the input UV laser pulse energy, with an efficiency of QE. The QE of copper cathodes varies between $10^{-6}$ to $10^{-4}$, which depends on the surface roughness, the presence of contaminants, and the field applied. In this $X$-band gun, the copper cathode has a relatively low QE of $2 \times 10^{-5}$. It requires $100 \mu \mathrm{J}$ UV pulse energy to generate electron charge of $420 \mathrm{pC}$. Conventionally, UV pulses near $266 \mathrm{~nm}$ are usually obtained from sum-frequency generation between a $400 \mathrm{~nm}$ second harmonic generation (SHG) and a $800 \mathrm{~nm}$ fundamental from Ti:sapphire laser systems, where the conversion efficiency to the third harmonic is usually less than $15 \%$. In this third-harmonic generation (THG) process, the THG pulse energy is proportional to the intensity cube of $800 \mathrm{~nm}$ fundamental, and any amplitude fluctuation is amplified in this nonlinear process. Although the pulse energy can be stabilized by feedback, it would add complexity to the laser system. 
When the peak intensity of laser pulses is sufficiently high, the multiphoton photoemission process can occur. Three-photon emission from a copper cathode has been observed with a higher QE than the single-photon emission when incident intensity is higher than $10 \mathrm{GW} / \mathrm{cm}^{2}$, and its mean thermal emittance is similar to UV photoemission at $266 \mathrm{~nm}$ [9]. Two-photon photoemission from a copper cathode has not been demonstrated before, it only needs a frequency doubling crystal compared to the THG system [10], and requires a similar intensity threshold to reach the same QE compared to three-photon emission, so that it could be a complementary method of generating photoelectrons.

Here we present a two-photon photoemission process from $400 \mathrm{~nm}$ laser pulses on a copper cathode. The $400 \mathrm{~nm}$ laser pulses are obtained by frequency doubling $800 \mathrm{~nm}$ fundamental from a commercial Ti:sapphire laser system. Its $\mathrm{QE}$ exceeds the single-photon process when the incident laser intensity is larger than $300 \mathrm{GW} / \mathrm{cm}^{2}$. We have demonstrated generating $100 \mathrm{pC}$ bunch charge with $52 \mu \mathrm{J}$ incident laser pulse with laser beam size of $1 \mathrm{~mm}$ full width at half maximum (FWHM). The emitted charge scales with laser pulse energy squared, and is inversely proportional to the laser pulse duration at the same laser pulse energy.

\section{EXPERIMENTAL APPARATUS, RESULTS, AND DISCUSSION}

The experimental schematic is depicted in Fig. 1. The laser system is a commercial Ti:sapphire laser system. It consists of a Ti:sapphire oscillator (Tsunami, Spectra Physics) providing $20 \mathrm{~nm}$ bandwidth pulses at $800 \mathrm{~nm}$, and a Ti:sapphire regenerative amplifier (Elite, Coherent) which amplifies the pulse energy to $4 \mathrm{~mJ}$. The laser system has a repetition rate of $600 \mathrm{~Hz}$, but is picked up at $300 \mathrm{~Hz}$ by an acousto-optic modulator. Chirped laser pulses of 200 ps pulse duration are then transported to the accelerator inside a cement tunnel 20-m downstream by an imagerelaying system (lens 1 in Fig. 1). Mirror loss along the transport reduces the laser pulse energy down to $3 \mathrm{~mJ}$. At arrival on the optical table adjacent to the accelerator, chirped pulses are compressed via a four-path singlegrating compressor combined with a retroreflector. With $20 \mathrm{~nm}$ bandwidth, the pulse can be compressed down to 50 fs full width at half maximum (FWHM). Its pulse duration can be tuned by adjusting the path length between the retroreflector and grating, whose group velocity dispersion is $-5000 \mathrm{fs}^{2} / \mathrm{mm}$. The pulse energy is $2.2 \mathrm{~mJ}$ after the compressor.

Pulse durations of $800 \mathrm{~nm}$ at the output of the compressor were measured by a single-shot autocorrelator, the compressor actuator position vs pulse duration is plotted in Fig. 2(a), the green curve is the theoretical calculation based on different group velocity dispersions, and it agrees well with the data. Some sample autocorrelation traces are

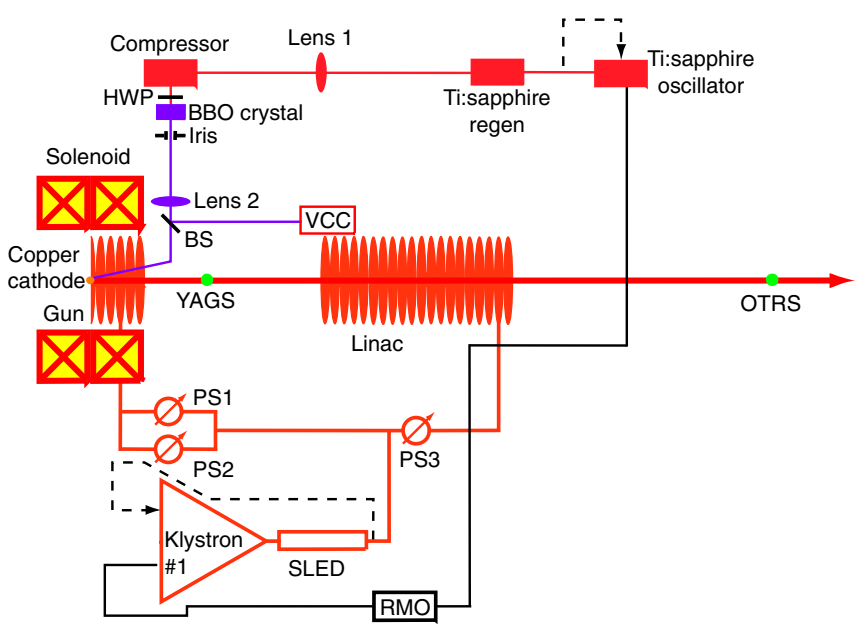

FIG. 1. Experiment schematic of the two-photon emission from a copper cathode of an $X$-band photoinjector (not to the scale). Lens 1 is the imaging relaying lens, lens 2 images an iris onto the cathode. HWP, half-wave plate; BS, beam splitter; VCC, virtual charge-coupled device camera; PS, phase shifter; RMO, radio master clock; YAGS, yttrium aluminum garnet screen; OTRS, optical transition radiation screen; PS, phase shifter; SLED, SLAC linac energy doubler. The solenoid has the bucking coil that makes sure the magnetic field on the cathode is zero. Klystron \#1 supplies energy to SLED, which doubles the energy and lowers the repetition rate of the klystron output pulses. PS1 and PS2 adjust the amplitudes and phases of SLED output if pulses to the gun, PS3 adjusts the phase of SLED output rf phases to the linac. YAGS collects charge on it, and is used to phase the 5.5 cell $X$-band rf gun. RMO provides the master radio clock to the laser oscillator and the klystron, which are both synchronized to the timing of the RMO.

shown in Fig. 2(b). These $800 \mathrm{~nm}$ fundamental wavelength pulses go through a $500 \mu \mathrm{m}$ thick type-I beta barium borate (BBO) crystal $(\theta=29.2$ degree), and are frequency doubled to $400 \mathrm{~nm}$. The phase matching bandwidth of this $500 \mu \mathrm{m}$ SHG BBO crystal is $10 \mathrm{~nm}$, which is lower for $20 \mathrm{~nm}$ of transform limited $50 \mathrm{fs}$ FWHM pulse at $800 \mathrm{~nm}$. The $10 \mathrm{~nm}$ lower bandwidth would elongate the pulse, but most of the energy is still in the main lobe, therefore, we still treat the $400 \mathrm{~nm}$ pulse duration as $1 / \sqrt{2}$ of the $800 \mathrm{~nm}$ fundamental pulses in the later part of this paper. A halfwave plate (HWP) before the BBO crystal rotates the input polarization of the $800 \mathrm{~nm}$ fundamental, it changes the phase match condition, and adjusts the laser power. Two dichroic mirrors reflect $400 \mathrm{~nm}$ second-harmonic generation (SHG), transmit $800 \mathrm{~nm}$ fundamental, and pulse energy of the SHG is up to $0.9 \mathrm{~mJ}$ when the system is optimized. Then the laser beam overfills an adjustable iris, it is reflected by two metal mirrors, and goes through a $75 \mathrm{~cm}$ (later changed to $100 \mathrm{~cm}$ ) focal length planoconvex lens (lens 2 in Fig. 1) to form a 2:1 (3:1) image on the copper cathode. A flip mirror can be inserted in the laser beam path to reflect the laser beam on a power meter to measure laser pulse energy. The laser beam is reflected by a 

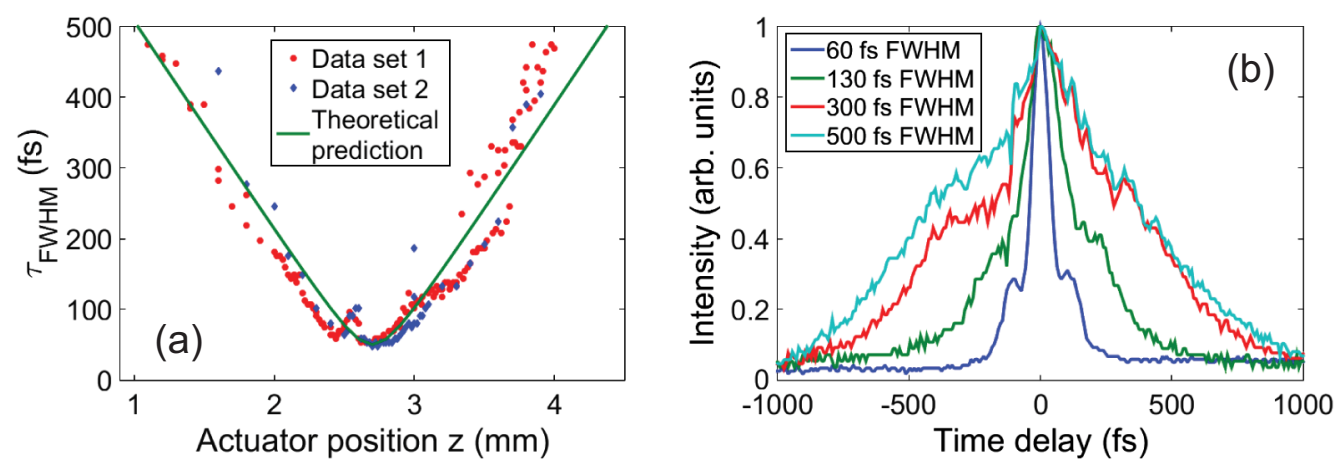

FIG. 2. Measured laser pulse durations and autocorrelations at $800 \mathrm{~nm}$. (a) Compressor actuator position vs pulse duration in FWHM, dots are experimental data, and the green curve is fitted by a model based on group velocity dispersion of the single-grating compressor. (b) Sample autocorrelation traces for pulse duration of 60, 130, 300, and $500 \mathrm{fs}$ FWHM.

$50 / 50$ beam splitter (later a dielectric mirror at $400 \mathrm{~nm}$, in order to have more laser pulse energy on the cathode), part of it then enters the rf gun enclosure, and the other part goes into a virtual cathode, a charge-coupled device camera after sufficient attenuation. The pulse energy before the entrance vacuum window is up to $0.3 \mathrm{~mJ}(0.6 \mathrm{~mJ})$ when laser system parameters are maximized. The vacuum window and the vacuum injection mirror are measured to have efficiency of 0.7 , and the laser energy on the cathode is up to $0.2 \mathrm{~mJ}$ $(0.4 \mathrm{~mJ})$. The incident angle of the laser beam on the cathode is approximately $3^{\circ}$. The surface of the copper cathode has a R of 0.48 at $400 \mathrm{~nm}$ [11], and half of the light is reflected downstream the linac beam line. In the later part of this paper, when calculating the QE, unless otherwise specified, we only count photons that are not reflected from the surface of the cathode, whose pulse energy is $E(1-R)$.

The emittance is calculated from beam size measurements on an optical transition radiation (OTR) screen, varied by scanning the strength of a quadrupole magnet upstream of the OTR screen. The reflection of $400 \mathrm{~nm}$ SHG laser beam goes downstream to the OTR screen, and optical low-pass filters are installed to filter out the $400 \mathrm{~nm}$ for those quad-scan emittance measurements.

The $X$-band rf gun has 5.5 cells, it can provide up to $200 \mathrm{MV} / \mathrm{m}$ peak field gradient at the cathode, and it normally operates at 50 degree injection phase in this experiment. If not mentioned otherwise, the rf field value is the peak field value, and the operating point is 50 degrees from the zero crossing. The higher peak field on the cathode can freeze the Coulomb forces between electrons, and preserve the thermal emittance in the photoemission process. The performance of this $X$-band photoinjector was elaborated and discussed in [12]. In a $n$-photon photoemission process, the emitted current density and laser intensity at photon energy $h v$ can be described by the Fowler-Dubridge theory [13-16],

$J_{n}(h v)=a_{n} A\left(\frac{e}{h v}\right)^{n}\left(1-R_{v}\right)^{n} I^{n} T_{e}^{2} F\left(\frac{n h v-e \varphi}{k_{b} T_{e}}\right)$, where $a_{n}$ is a constant related to the likelihood of $n$-photon photoemission, $A=120 \mathrm{~A} / \mathrm{cm}^{2} \mathrm{~K}^{2}$ is the Richardson constant, $e$ is the electron charge, $R_{v}$ is the reflectivity of optical frequency $v, I$ is the laser intensity, $T_{e}$ is the temperature on the surface of the cathode, $k_{b}$ is Boltzmann's constant, $\varphi$ is the work function of the cathode, $F(x)=\int_{0}^{\infty} \ln \left(1+e^{-y+x}\right) d y$ is the Fowler function. In our experiment, $J_{2}(h v)$ stands for the current of two-photon emission with photon wavelength at $\mathrm{nm}$, and $J_{1}(3 / 2 h v)$ stands for the current of single-photon emission at UV wavelength of $266 \mathrm{~nm} . J_{2}(h v)$ becomes larger than $J_{1}(3 / 2 h v)$ when laser intensity is larger than a critical value $I_{\mathrm{cr}}$, given by Eq. (2), this two-photon process has a higher electron yield compared to the single-photon photoemission process:

$$
I_{\mathrm{cr}}=\frac{\frac{2}{3} a_{1}\left(1-R_{3 / 2 v}\right) F\left(\frac{3 / 2 h v-e \varphi}{k_{b} T_{e}}\right)}{a_{2}\left(1-R_{v}\right)^{2} F\left(\frac{2 h v-e \varphi}{k_{b} T_{e}}\right)} \frac{h v}{e} .
$$

It was measured that $a_{1} \sim 10^{-14} \mathrm{~cm}^{2} \mathrm{~A}^{-1}[15]$ and $a_{2} \sim$ $10^{-25} \mathrm{~cm}^{4} \mathrm{~A}^{-2}$ [16], and assuming that the blue $(400 \mathrm{~nm})$ and UV $(266 \mathrm{~nm})$ light have reflectivity of 0.48 and 0.33 respectively, and temperature of electrons at several thousand $K$ [17], we calculate the critical power to be $I_{\text {cr }} \sim 100 \mathrm{GW} / \mathrm{cm}^{2}$. The highest peak intensity in our experiment is $80 \mathrm{GW} / \mathrm{cm}^{2}$, that is smaller than the damage threshold of copper [18]. However, it is also known that in the presence of the $\mathrm{MV} / \mathrm{m}$ electric field, the damage threshold of copper could be 1 or 2 orders magnitude lower. Furthermore, at the laser pulse duration 40 fs FWHM at $300 \mathrm{~Hz}$, at the critical power, the average fluence corresponds to $1.2 \mathrm{~J} / \mathrm{cm}^{2}$, which is comparable to the ablation threshold of copper in air [19,20]. Most of the time, pressure of the gun vacuum is usually $10^{-9}$ torr. Thus, there was no immediate observable damage to the cathode. Ee did observe the decrease of the QE after eight hours. Processing of the gun several hours with rf power and without the laser beam can remove the contamination 

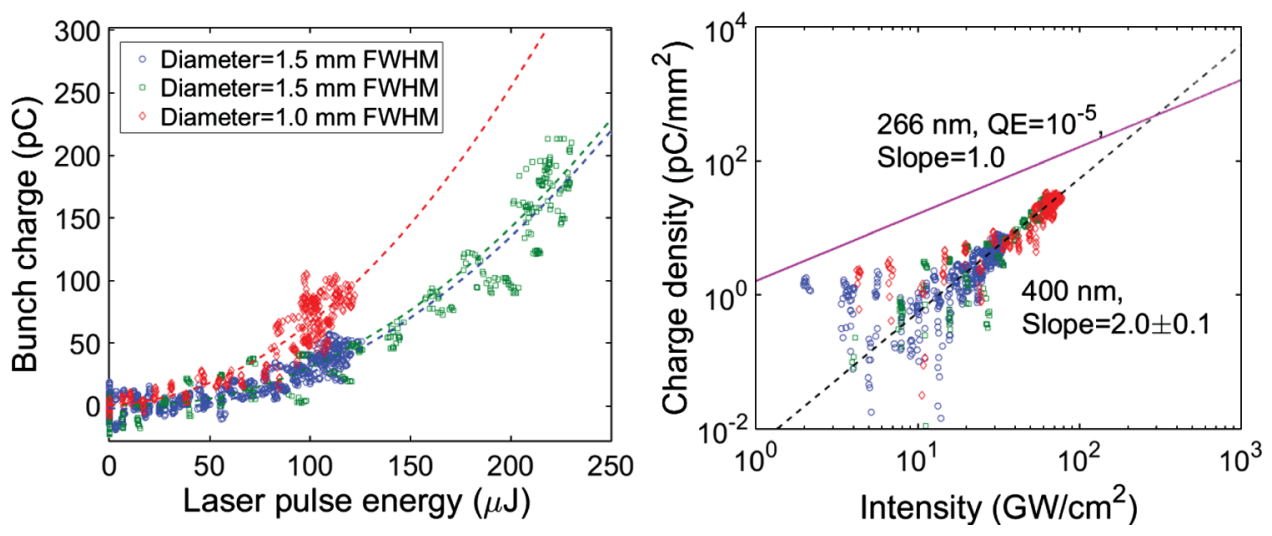

FIG. 3. Electron bunch charge and absorbed laser intensity. (a) Incident laser pulse energy E vs electron bunch charge. (b) Incident laser intensity I vs electron charge density. The large scatter of data points mainly comes from the time jitter between the laser pulses and rf field inside the gun, it also comes from the amplitude and phase noise from the rf field itself. Both of them can change the instant field at the cathode, and change the QEs, thus the charges of electron bunches. Because this is an $X$-band photoinjector, therefore it is more sensitive to the phase noise compared to the traditional S-band photoinjectors. Meanwhile, the jittering of rf field changes the effective work function of the copper cathode, and also contributes to the large scatter of data in Figs. 3 and 4.

and recover the QE. Previous work has also demonstrated that the overall laser-induced damage threshold is higher at longer wavelength because of the lower effective cross section in the multistep multiphoton process [21].

We measured the laser power vs emitting charge, the laser power is measured on a power meter, and electron charge is collected by a Faraday cup just after the gun. In the experiment, we rotate a HWP before the BBO crystal to adjust the laser power. In Fig. 3(a), for blue circled data points, the last optic before the vacuum is a 50/50 beam splitter, and the beam spot size is $1.5 \mathrm{~mm}$ FWHM on the cathode; for green square and red diamond data points, this beam splitter is replaced by a dielectric mirror, and the beam sizes are 1.5 and $1 \mathrm{~mm}$ FWHM on the cathode respectively. It is apparent that electron charge scales as the square of laser pulse energy, as expected from a two-photon photoemission process. The dashed curves are quadratic fits of the data to guide the eye. Figure 3(b) plots the laser intensity vs electron charge density. The curve has a slope of $2.0 \pm 0.1$, which confirms that the emitted charge is generated from a two-photon photoemission process. The curve of single-photon photoemission from UV at $266 \mathrm{~nm}$ with $\mathrm{QE}$ of $10^{-5}$ is also plotted on the same graph, it crosses the two-photon photoemission curve at a laser intensity of $I_{\text {cr }} \sim 300 \mathrm{GW} / \mathrm{cm}^{2}$. The QE is relatively low when intensity is below the critical intensity $I_{\mathrm{cr}}$, it reaches $10^{-5}$ at $I_{\mathrm{cr}}$, and exceeds the typical single-photon QE of $10^{-5}$ when intensity is larger than $I_{\mathrm{cr}}$. It is worth noting that the copper cathode has a reflectivity of 0.48 for $400 \mathrm{~nm}$ as reported in [11], thus, in order to utilize this high QE of the two-photon photoemission process, antireflection coating is required on the surface of the copper cathode.

We also measured the charge at different rf field gradients on the surface of the cathode. Higher gradient lowers the effective work function $\varphi$ to $\varphi-\sqrt{\frac{e \beta E_{0} \sin \theta}{4 \pi \epsilon_{0}}}$, and makes the photoemission process more efficient, where $\beta$ is the enhancement factor, $E_{0} \sin \theta$ is the rf field at injection phase $\theta, \epsilon_{0}$ is the absolute permittivity. We measured the photoemission at the field gradient of 140 and $200 \mathrm{MV} / \mathrm{m}$ as shown in Fig. 4, the enhancement factor is 1.5 in this experiment by fitting the data. Although higher field gradients increase dark currents, it increases the $\mathrm{QE}$ as well. The QE at $200 \mathrm{MV} / \mathrm{m}$ is substantially higher compared to its value at $140 \mathrm{MV} / \mathrm{m}$.

We studied the photoemission at different input laser pulse durations as well. The charge emitted from a photocathode can be described by the following equation:

$$
Q=J \tau A=C I^{2} \tau A=C \frac{E^{2}}{\tau A}
$$

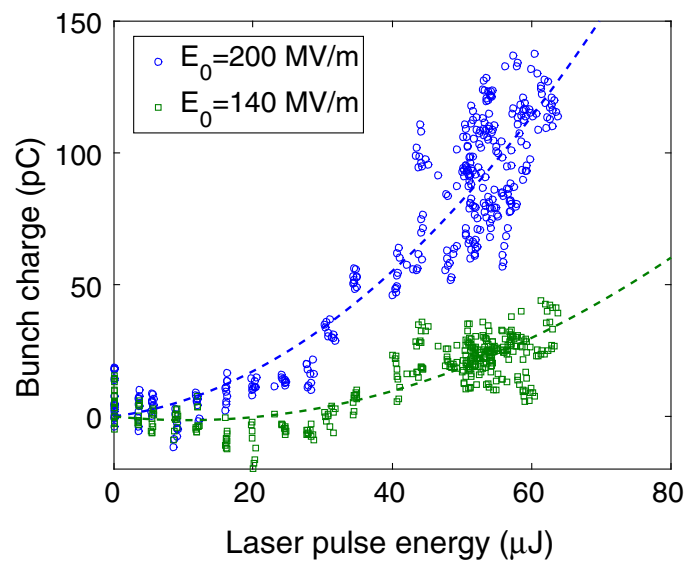

FIG. 4. Absorbed laser pulse energy E(1-R) vs electron bunch charge. The dashed lines are quadratic fits of the data. The $E_{0}$ is the peak field, and the gun operates at 50 degrees from zero crossing. The reason for large scatter of data points is the same as in Fig. 3. 

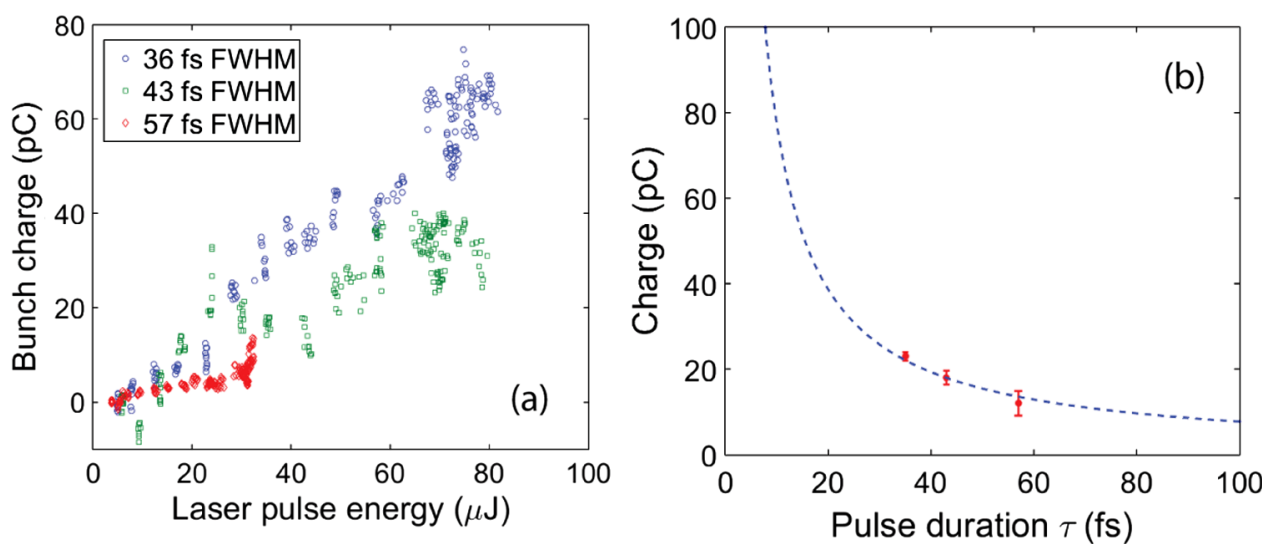

FIG. 5. Laser pulse duration and electron bunch charge. (a) Absorbed laser pulse energy E(1-R) vs electron bunch charge at different pulse duration. (b) Laser pulse duration vs electron bunch charge at $30 \mu \mathrm{J}$ laser pulse energy, the dashed line is the fit of $1 / \tau$, the error bars are calculated from Fig. 5(a).

where $Q$ is the emitted charge, $J$ is the charge current on the surface, $\tau$ is the laser pulse duration, $A$ is the area of the laser spot area, $I$ is the laser intensity, $E$ is the laser pulse energy. Therefore, the charge is inversely proportional to the pulse duration. The pulse duration of the $800 \mathrm{~nm}$ fundamental can be tuned by adjusting path length in the pulse compressor, and the $400 \mathrm{~nm}$ SHG pulse duration is assumed to have $1 / \sqrt{2}$ of the pulse duration as the $800 \mathrm{~nm}$ fundamental pulses, when the group velocity mismatch inside the $500 \mu \mathrm{m}$ $\mathrm{BBO}$ crystal is negligible. We measured the bunch charge at different pulse durations with beam size $\sigma$ of $0.4 \mathrm{~mm}$. For the same pulse energy, shorter pulse duration has higher QE, because of its higher peak intensity. The data are depicted in Fig. 5; Fig. 5(a) shows the laser pulse energy vs bunch charge at laser pulse duration $(266 \mathrm{~nm})$ of 36,43 , and $57 \mathrm{fs}$ FWHM. Figure 5(b) shows the laser pulse duration vs bunch charge at $35 \mu \mathrm{J}$ pulse energy; the data was derived from Fig. 5(a) where the pulse energy is $35 \mu \mathrm{J}$. At this pulse energy, the emitted charge does scale inversely proportional to pulse duration $\tau$, as expected from Eq. (3). From the data, it is calculated that $C=5 \times 10^{-22} \mathrm{~A} \mathrm{~m}^{2} / \mathrm{W}^{2}$, which corresponds to $a_{2}=10^{-25} \mathrm{~cm}^{4} / \mathrm{A}^{2}$.

We have also measured the emittance at very low bunch charges as the thermal emittance from the copper cathode

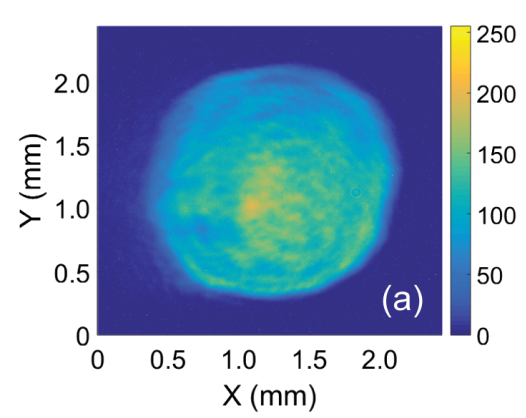

downstream the linac at $70 \mathrm{MeV}$ electron beam energy; the result is presented in Fig. 6. Figures 6(a) and 6(b) depict a typical laser beam spatial profile and laser beam size vs emittance at very small charge respectively. Electron beam size is measured on an OTR screen at various quadruple strengths, and emittance is calculated from the transfer matrix. Thermal emittance is given by $\epsilon_{n}=\sigma \sqrt{\frac{M T E}{m_{e} c^{2}}}=\sigma \sqrt{\frac{2 h v-e \varphi}{m_{e} c^{2}}}$, where $\sigma$ is laser beam size (assuming that the spatial distribution obeys normal distribution $\left.N(0, \sigma) \sim \frac{1}{\sigma \sqrt{2 \pi}} e^{-\frac{\rho^{2}}{2 \sigma^{2}}}\right), M T E$ is the mean thermal energy, $h v$ is the photon energy, $\varphi$ is the work function, $e$ is the electron charge, $m_{e}$ is the electron mass, and $c$ is the speed of light.

The measured thermal emittance is approximately $2.7 \mathrm{~mm} \mathrm{mrad} / \mathrm{mm}$, which is 60 percent larger compared to the theoretical predictions $1.7 \mathrm{~mm} \mathrm{mrad} / \mathrm{mm}$, similar to the case of single-photon emission at $266 \mathrm{~nm}$, and threephoton emission at $800 \mathrm{~nm}$ [9]. The large value of the thermal emittance can be understood by the poor surface uniformity of the cathode. After two years from commissioning, the cathode surface was noticeably damaged. Both rf breakdowns and too high fluence of short laser pulses generated ripple patterns and scratches on the cathode

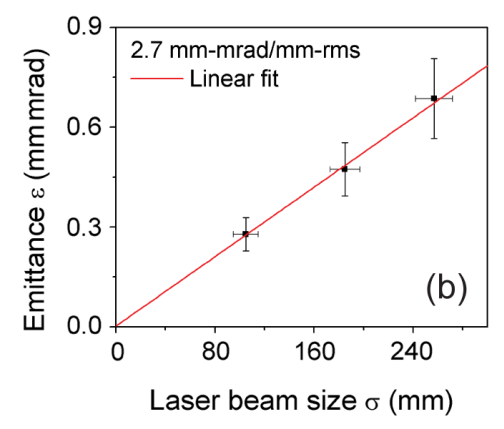

FIG. 6. Laser beam spatial profile (a) and beam size vs emittance (b) at very low bunch charge. The red line is linear fit of the data. 
surface. As electric field is normal to the surface of those irregular features, the transverse momentum spread increases, the emittance is $2.7 \mathrm{~mm}$-mrad per rms $\mathrm{mm}$.

We have demonstrated the two-photon photoemission on a copper cathode in an $X$-band photoinjector. The $\mathrm{QE}$ of the two-photon photoemission exceeds the single-photon process at a laser intensity of $300 \mathrm{GW} / \mathrm{cm}^{2}$, the electron charge yield scales inversely with laser pulse duration at the same laser pulse energy. The two-photon photoemission requires a simpler laser system over a conventional UV laser system, and could be useful for future photoinjector applications.

\section{ACKNOWLEDGMENTS}

We thank Bubby McKee for his help in the experiment. This work was partially supported by the U.S. Department of Energy.

[1] C. N. Berglund and W. E. Spicer, Photoemission studies of copper and silver: Theory, Phys. Rev. A 136, A1030 (1964).

[2] T. Rao and D. Dowell, An engineering guide to photoinjectors, http://arxiv.org/ftp/arxiv/papers/1403/1403.7539 .pdf.

[3] G. Farkas, A. Kohazi-Kis, and C. Toth, Above-threshold multiphoton photoelectric effect of a gold surface, Opt. Eng. 32, 2476 (1993).

[4] A. Damascelli, G. Gabetta, A. Lumachi, L. Fini, and F. Parmigiani, Multiphoton electron emission from $\mathrm{Cu}$ and W: An angle-resolved study, Phys. Rev. B 54, 6031 (1996).

[5] R. Akre, D. Dowell, P. Emma, J. Frisch, S. Gilevich, G. Hays, Ph. Hering, R. Iverson, C. Limborg-Deprey, H. Loos, A. Miahnahri, J. Schmerge, J. Turner, J. Welch, W. White, and $\mathrm{J}$. Wu, Commissioning the Linac Coherent Light Source injector, Phys. Rev. ST Accel. Beams 11, 030703 (2008).

[6] M. Trovo et al., Status of the FERMI@Elettra Photoinjector, Proceedings of the 11th European Particle Accelerator Conference, Genoa, 2008 (EPS-AG, Genoa, Italy, 2008), MOPC080.

[7] F. Sannibale et al., Advanced photoinjector experiment photogun commissioning results, Phys. Rev. ST Accel. Beams 15, 103501 (2012).

[8] T. Hara, K. Togawa, and H. Tanaka, The 34th International Free-Electron Laser Conference (FEL2012), Nara, Japan, 2012, (2012), МОOВ03.
[9] P. Musumeci, L. Cultrera, M. Ferrario, D. Filippetto, G. Gatti, M. S. Gutierrez, J. T. Moody, N. Moore, J. B. Rosenzweig, C. M. Scoby, G. Travish, and C. Vicario, Multiphoton Photoemission from a Copper Cathode Illuminated by Ultrashort Laser Pulses in an rf Photoinjector, Phys. Rev. Lett. 104, 084801 (2010).

[10] L. Kasmi, D. Kreier, M. Bradler, E. Riedle, and P. Baum, Femtosecond single-electron pulses generated by twophoton photoemission close to the work function, New J. Phys. 17, 033008 (2015).

[11] See, for example, W. M. Rohsenow and H. Choi, Heat Mass and Momentum Transfer (Prentice-Hall, New York, 1961); Handbook of Chemistry and Physics, 96th ed. (CRC Press, Boca Raton, Florida, 2015).

[12] C. Limborg-Deprey, C. Adolphsen, D. McCormick, M. Dunning, E. Hemsing, K. Jobe, H. Li, T. Raubenheimer, A. Vrielink, F. Wang, and S. Weathersby, An $X$-band photoelectron rf gun for an ultrafast electron diffraction source (to be published).

[13] R. H. Fowler, The analysis of photoelectric sensitivity curves for clean metals at various temperatures, Phys. Rev. 38, 45 (1931).

[14] L. E. Dubridge, Theory of the energy distribution of photoelectrons, Phys. Rev. 43, 727 (1933).

[15] T. Tsang, T. Srinivasan-Rao, and J. Fischer, Surfaceplasmon field-enhanced multiphoton photoelectric emission from metal films, Phys. Rev. B 43, 8870 (1991).

[16] J. H. Bechtel, W. L. Smith, and N. Bloembergen, Twophoton photoemission from metals induced by picosecond laser pulses, Phys. Rev. B 15, 4557 (1977).

[17] J. G. Fujimoto, J. M. Liu, E. P. Ippen, and N. Bloembergen, Femtosecond Laser Interaction with Metallic Tungsten and Nonequilibrium Electron and Lattice Temperatures, Phys. Rev. Lett. 53, 1837 (1984).

[18] R. W. Boyd, Nonlinear Optics, 3rd ed. (Academic Press, New York, 2012).

[19] M. Hashida, A. F. Semerok, O. Gobert, G. Petite, Y. Izawa, and J. F-Wagner, Ablation threshold dependence on pulse duration for copper, Appl. Surf. Sci. 197-198, 862 (2002).

[20] P. T. Mannion, J. Magee, E. Coyne, G. M. O'Connor, and T. J. Glynn, The effect of damage accumulation behavior on ablation thresholds, and damage morphology in ultrafast laser micro-machining of common metals in air, Appl. Surf. Sci., 233, 275 (2004).

[21] C. W. Carr, H. B. Radousky, and S. G. Demos, Wavelength Dependence of Laser-Induced Damage: Determining the Damage Initiation Mechanisms, Phys. Rev. Lett. 91, 127402 (2003). 